\title{
Brain Tumor Segmentation in MRI Images
}

\author{
Adarsh Dhiman $^{1}$, B.S. Satpute ${ }^{2}$
}

1(Department of computer engineering, Dr. D. Y. Patil Institute of TechnologyPimpri, Pune-41118, India)

2(Department of computer engineering, Dr. D. Y. Patil Institute of TechnologyPimpri, Pune-41118, India)

\section{Abstract:}

Human brain is complex thing and identifying a disease at earlier stages is one of the difficult tasks in medical field. The identification; manual segmentation and detection of infected areas in brain MRI images are a tedious and time-consuming task. To reduce this time constraint; neural networks are an ideal in recognizing diseases using scans since there is no need to provide a specific algorithm on how to identify the disease.

In earlier years of machine learning the features were designed manually by the domain expert and it required deep understanding and domain specific knowledge to carry out this task. In recent years there are tremendous developments in the field of machine learning especially deep learning. Deep learning made it possible to learn the hierarchical features present in the data. The idea is to use this knowledge in the medical field as well to learn these features spontaneously and automatically segment the MRI images.

In this paper, we review brain tumor segmentation in MRI images. In due course, we review the different techniques to segment the MRI images. We discuss as well the challenges involved in these segmentation techniques, and their potential in the future.

Keywords - Brain Tumor, Deep Learning, Image Segmentation, Convolutional Neural Network (CNN).

\section{INTRODUCTION}

Over last few decades the advancements in the medical imaging techniques like MRI has caused earlier diagnosis and prognosis of the disease. This data is analysed manually by the radiologist to find the disease. It is really tiresome job to manually analyse such huge corpus of images without failing. To overcome this difficulty of manual interpretation of data there is need to bring automation to this task. To this cause different medical institutes are making the medical data available freely like BraTS dataset which is helping independent researchers as well to carry out their studies on MRI image segmentation and computer aided detection. This digitization lead revolution of medical data has resulted in huge volume of data. The problem with such huge corpus of information is that it is really overwhelming task to analyse and find results from this data. There is utmost need to apply deep learning techniques to this volume of data to interpret and analyse results automatically with lesser error rates.

The deep learning approach can be used to extract salient data features which in turn mean brain health which will help practitioners to take better decisions. Machine learning helps to extract hierarchical data features from MRI images which help to create computational model that provides the insight to the data. The complex relationships explored by the model are sometimes not even obvious to the radiologist. These hierarchical features are self-learned by the model.

There is need to use deep learning techniques in medical image analysis in order to extract salient data features for improved diagnosis and prognosis of diseases.

There is need to use deep learning techniques in medical image analysis in order to extract salient data features for improved diagnosis and prognosis of diseases. 


\section{LITERATURE SURVEY}

\section{A. U-Net Based Deep Convolutional Networks for Brain MRI Segmentation:}

In this paper, we focus on how we can leverage UNet based Convolutional Neural Networks to deal with variable edge problem of the imaged object (brain tumor). The skip architecture is used to handle variable edge problem. This method has shown promising results on MRI images as well. In this study the authors have used U-Net based CNN as a base model. This scheme has obtained good results for the core tumor segmentation.

\section{B. Stacked De-noising Auto-Encoder Deep Learning Framework:}

In this paper, a novel approach which is based on the concept of deep learning, has been proposed to address the challenges like human intervention, domain knowledge etc. to segment brain tumors. This method first extracts image patches from medical images and treats the gray level of image patches as the input to the deep learning framework. The Stacked De-noising Auto-Encoder (SDAE), which is one of the deep learning frameworks, automatically learns the high level latent representation for future classification. Then, the classification result is mapped into a binary image and finally, the post-processing step is adopted to achieve the final segmentation result.

\section{Deep Learning in Medical Image Analysis:}

In this paper, different deep learning neural networks like Feed-Forward Neural Networks, Convolutional Neural Networks etc. are discussed with their application in the medical Image Analysis for automatic segmentation and Computer Aided Detection.

\section{Image Segmentation Strategies and CNN:}

This paper discusses, the different principle ways which deal with semantic division like pixel-wise division, where a little fix of a picture is utilized to order the middle pixel, and completely convolutional designs where the system input is the full picture and yield is a semantic division volume. This study utilized Convolutional Neural Network for medical image segmentation.

\section{E. Brain Tumor Segmentation using Convolutional Neural Network:}

This paper, proposes a CNN-based method for segmentation of brain tumors in MRI images. In this proposal the pre-process stage consists of bias correction and patch normalization. In order to increase low-grade glioma (LGG) classes the training patches are rotated to produce new LGG classes. The deep CNN architecture is built using $3 \mathrm{X} 3$ kernels over convolutional layers.

\section{PROPOSED METHODOLOGY}

In this project, the U-Net based fully Convolutional Neural Network is proposed to carry out the brain MRI segmentation.

In this system user interacts to provide the target image for segmentation.

\section{A. System Architecture:}

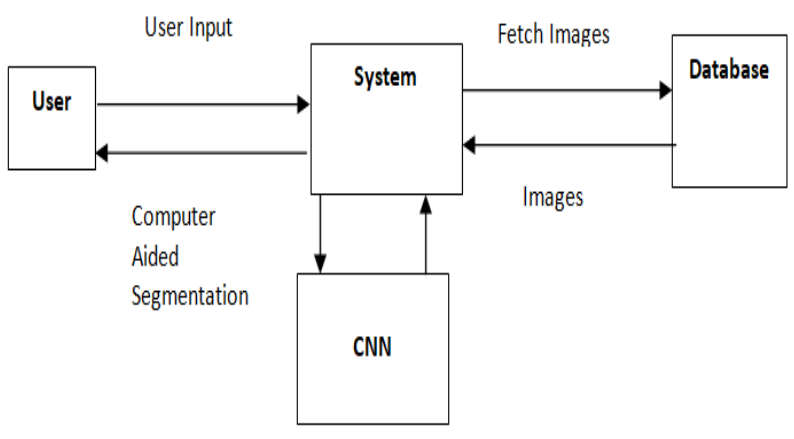

Fig 1. Overview of System Architecture

\section{B. Data Collection:}

The proposed method will use the images available in the BRATS 2015 datasets, which has high grade glioma (HGG) as well as rare low-grade glioma (LGG) MRI scans. Also, the multimodal MRI images which are T1-weighted (T1), T2-weighted (T2) and FLAIR are available for each patient. 


\section{Network Architecture Design:}

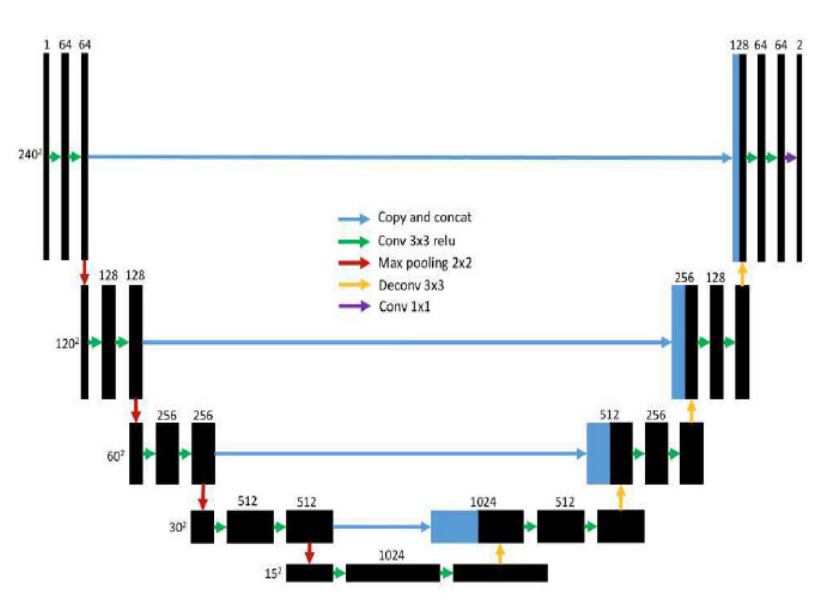

Fig. 2. U-Net based CNN Architecture

The proposed network architecture, which is based on the U-Net, has a contraction path and an expansion path as shown in Fig. 2. The contraction path has four blocks. Each block has 3X3 convolutional layers and ReLU activation. In case of contraction path, $2 \times 2$ Max Pool is applied to the end of every block, to reduce the size of feature map. The expansion path has also four blocks. Each of the blocks in the expansion path has deconvolutional layer, a link to the corresponding feature map in the contraction path and 3X3 convolutional layers and ReLU activation.

\section{Evaluation Function:}

The segmentation result of the $\mathrm{CNN}$ will be evaluated based on the dice similarity coefficient (DSC) which gives the ratio between the manually labelled brain tumors by radiologist and the segmentation results of the deep convolutional neural network. The DSC and sensitivity are calculated as follows:

$$
\begin{aligned}
& \mathrm{DSC}=\frac{2 \mathrm{TP}}{\mathrm{FP}+2 \mathrm{TP}+\mathrm{FN}} \\
& \text { Sensitivity }=\frac{\mathrm{TP}}{\mathrm{TP}+\mathrm{FN}} .
\end{aligned}
$$

In the above formula the abbreviations are denoted as follows:

TP stands for True Positive.

FP stands for False Positive.

FN stands for False Negative.

\section{RESULT AND DISCUSSIONS}

In this study the proposal is to use U-Net based fully convolutional neural network for brain image segmentation. Deep learning or deep convolutional neural networks are providing high performances in medical image analysis compared to other techniques based on DSC.

The network will be provided with the input MRI image and output will be the segmented image.

\section{Input Image:}

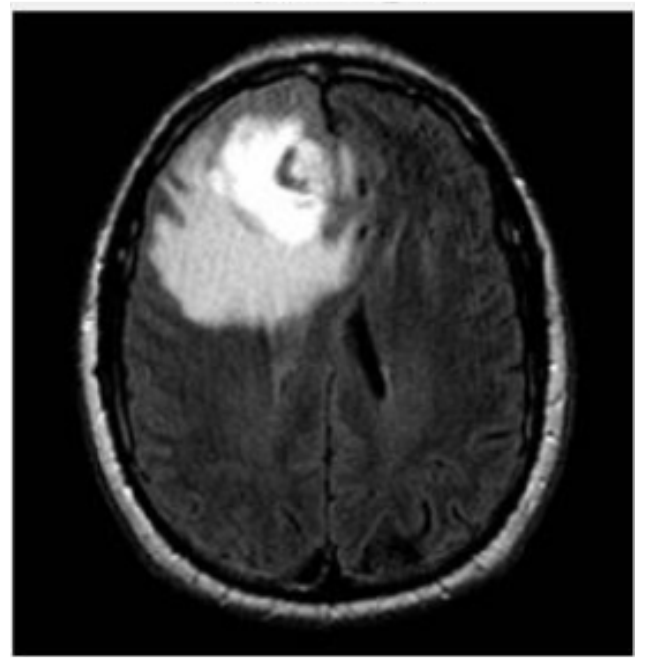




\section{Segmented Image:}

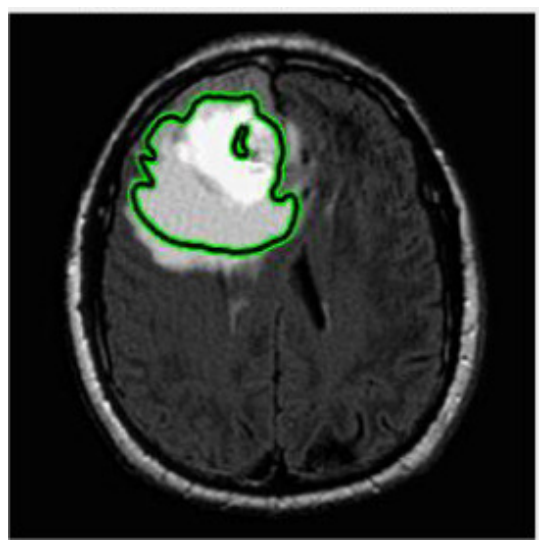

This network can be further used to study all the modalities available in the MRI images to prove the validity and range of network to segment brain images.

\section{CONCLUSIONS}

This study proposes use of CNN based learning techniques to segment the brain MRI images in medical field. The advancement in the segmentation techniques will greatly assist in the computer aided detection of tumour and hence the practitioners in the medical science. Recent progress in deep learning has enabled to discover the cellular features in the images merely from data. Deep learning methods have achieved phenomenal results across different medical applications like Alzheimer's disease, Pneumonia etc.; however, there is still room for improvement.

\section{REFERENCES}

1. Hao Dong, Guang Yang, Fangde Liu, Yuanhan Mo, YikeGuo. Data Science Institute, Imperial College London, SW7 2AZ, London, UK. 'Automatic Brain Tumor Detection and Segmentation Using U-Net Based Fully Convolutional Networks'.

2. Zhe Xiao, Ruohan Huang, Yi Ding, TianLan, RongFeng Dong and Zhiguang Qin, School of Infonnation and Software Engineering,University of Electronic Science and Technology of China. 'A
Deep Learning-Based Segmentation Method for Brain Tumor in MR Images'.

3. Shen, Guorong Wu and Heung-Il Suk, Department of Radiology, University of North Carolina, Chapel Hill, North Carolina 27599. 'Deep Learning in Medical Image Analysis'.

4. Manda SSSNMSRL Pavan, P. Jagadeesh,Department of Electronics and Communication Engg,Saveetha School of Engineering. International Journal of Pure and Applied Mathematics, 'BRAIN TUMOR SEGMENTATION USING COVOLUTIONAL NEURAL NETWORK IN MRI IMAGES'.

5. Sérgio Pereira, Adriano Pinto, Victor Alves, and Carlos A. Silva, IEEE TRANSACTIONS ON MEDICAL IMAGING, VOL. 35, NO. 5, MAY 2016. 'Brain Tumor Segmentation Using Convolutional Neural Networks in MRI Images'.

6. HapsariPeni Agustin Tjahyaningtijas 2018 IOP Conf. Ser.: Mater. Sci. Eng. 336 012012. 'Brain Tumor Image Segmentation in MRI Image'.

7. Adnan Qayyuma, Syed Muhammad Anwar, Muhammad Majid, Muhammad Awais, MajdiAlnowamid, CVSSP, University of Surrey, Surrey, UK. 'Medical Image Analysis using Convolutional Neural Networks: A Review'.

8. Kavitha Angamuthu Rajasekaran and Chellamuthu Chinna Gounder, DOI: 10.5772/intechopen.71416. 'Advanced Brain Tumor Segmentation from MRI Images'. 